This document is the unedited Author's version of a Submitted Work that was subsequently accepted for publication in J ournal of Physical Chemistry C, copyright (c) American Chemical Society after peer review. To access the final edited and published work see: https://dx.doi.org/10.1021/acs.jpcc.8b09774. 


\section{Thermal control of intermolecular interactions and tuning of fluorescent state energies}

Giuseppina Massaro, ${ }^{\dagger}{ }^{+}$Dani Ruiz-Molina, ${ }^{\#}$ Jordi Hernando, ${ }^{+}$Claudio Roscini, ${ }^{\# *}$ Loredana Latterini. $^{+^{* *}}$

† Department of Chemistry, Biology and Biotechnology, Perugia University, Via Elce di sotto, 8, 06123 Perugia, Italy

\# Catalan Institute of Nanoscience and Nanotechnology (ICN2), CSIC and BIST, Campus UAB, Bellaterra, 08193 Barcelona, Spain.

+ Departament de Química, Universitat Autònoma de Barcelona, Edifici C/n, Campus UAB, 08193 Cerdanyola del Vallès, Spain.

† Present address: Institute of Chemical Research of Catalonia (ICIQ), Avda. Països Catalans 16, 43007 Tarragona, Spain

\section{Corresponding Authors}

* Loredana Latterini: loredana.latterini@unipg.it

* Claudio Roscini: croscini@icn2.cat. 


\begin{abstract}
The prospect to tune the energy of emitting states through external stimuli opens the possibility to shift the energy of emitting units on demand and control the bimolecular processes they are involved in. To prove this concept, the fluorescence properties of three differently 9,10substituted anthracene derivatives are investigated in a phase change material (eicosane). The liquid-to-solid transition of the medium leads to an increase of the local dye concentration, a shortening of the intermolecular distances and the establishment of excited and ground-state interactions. As a result, a new contribution to the overall luminescence derives from the downshifted emission (up to $0.7 \mathrm{eV}$ ) from excimer-like species is observed. The addition of a second dye (a Pt-porphyrin) reduces the efficiency of excited and ground-state complexes between fluorophore units, although does not prevent the formation of multichromophoric aggregates where interactions between Pt-porphyrin and the emissive state of anthracene derivatives are observed.
\end{abstract}

KEYWORDS. Phase change materials, fluorescence, aggregation, excimer, excited states energy modulation 


\section{Introduction}

In properly designed systems, the control of homochromophoric intermolecular distances or interactions can modify the nature of the lowest excited states, which affects the efficiency, and interestingly, the energy of emitted radiation. ${ }^{1,2}$ On the other hand, by controlling the energy of the luminescent state, the management of the rate and the efficiency of heterochromophoric bimolecular processes involving the excited states can be achieved. For instance, the luminescence originating from non-linear processes, such as the anti-Stokes emission of upconverting molecular materials, can be modulated in this way. In particular, triplet-triplet annihilation based upconversion (TTA-UC) requires intermolecular energy transfer among the excited states of donors and acceptors and these interactions can be manipulated by tuning the energy of their emitting states. This is valid not only for liquid solutions but also for solid materials, where the energy migration through molecular aggregates ${ }^{3,4}$ emerged as an alternative strategy to overcome the lack of molecular diffusion ${ }^{5,6}$ and allow TTA-UC in the solid phase.

We have previously exploited the aggregation phenomenon of the donor units in aliphatic phase change materials (PCMs) to achieve thermally switchable TTA-UC. ${ }^{7}$ While negligible energy transfer processes and, as such, red phosphorescence from the donors were observed in the liquid state of the system at relatively low dye concentrations, blue upconverted emission was registered upon solidification of the medium. This was due to chromophore aggregation in the solid PCM, which facilitated the energy transfer processes that underlie TTA-UC. Though we demonstrated the very efficient triplet-triplet energy transfer process between sensitizers and emitters $\left(\Phi_{\mathrm{ET}}>95 \%\right)$ in the solid PCM and the role of sensitizer aggregates, we did not explore the occurrence of interactions among the emitter units and their impact on the energy of their emitting state. 
Notably, the fluorescence of the emitters is able to report on intermolecular interactions occurring on the singlet state but also on the triplet manifold. For instance, bimolecular interactions or extended aggregation can stabilize singlet and triplet states thus fastening energy transfer or energy migration. These interactions can enhance the efficiency of triplet energy transfer (up to $\Phi_{T T E T} \sim 0.9$ ) involved in TTA-UC and explain the high UC quantum yield ( $\Phi_{U C} \sim$ $0.06)^{7}$ observed below the PCM melting point $\left(\mathrm{T}_{\mathrm{m}}\right)$. Furthermore, the outcome of careful analysis of the fluorescence data could be easily extended to many fundamental processes.

In view of this, we investigate herein the fluorescence properties of anthracene dyes (frequently used as emitters in TTA-UC) as a function of the PCM phase and we further extend these studies in the presence of 2,3,7,8,12,13,17,18-octaethyl-21H,23H-porphine platinum(II) (PtOEP), usually employed as an antenna to sensitize TTA-UC. The modifications of the fluorescence spectra and decay times should provide information on the ground and/or excited state intermolecular interactions and document changes of their singlet state energies, which have the same behaviour as the corresponding triplet states, according to literature data. ${ }^{8-11}$ To this aim, we select three anthracene derivatives with different 9,10-substitutents (Scheme S1); the 9,10 -substitutions can significantly alter the $\pi-\pi$ intermolecular interactions occurring in the ground and/or excited state but, according to experimental data and DFT calculations, only induce minimal changes of the energies either in the singlet and triplet manifold. Eicosane (EC), an aliphatic paraffin already used in our previous work, is chosen as a PCM $\left(\mathrm{T}_{\mathrm{m}}=37^{\circ} \mathrm{C}\right) .{ }^{12}$ 


\section{Experimental section}

Materials. 9,10-diphenylanthracene (DPA), 9,10-dimethylanthracene (DMA, 99\%, EGA Chemie), anthracene (ANT, 99\%, J. T. Baker), 2,3,7,8,12,13,17,18-octaethyl-21H,23H-porphine platinum(II) (PtOEP, 98\%, Sigma Aldrich) were used without further purification. Dichloromethane $(\mathrm{DCM}, \geq 99.5 \%)$ has been provided by Alfa Aesar. Eicosane (EC, 98\%, melting temperature, $\mathrm{Tm}=37^{\circ} \mathrm{C}$ ) was purchased from Merkel and used as received.

Solutions preparation. Stock solutions of DPA $\left([\mathrm{DPA}]_{0}=1.35 \mathrm{mM}\right), \mathrm{DMA}\left([\mathrm{DMA}]_{0}=3.5 \mathrm{mM}\right)$, ANT $\left([\mathrm{ANT}]_{0}=0.53 \mathrm{mM}\right)$ and PtOEP $\left([\mathrm{PtOEP}]_{0}=0.12 \mathrm{mM}\right)$ were prepared in dichloromethane and stored in the dark to prevent any photodegradation. The proper amount of the stock solution was added to the weighted quantity of $\mathrm{EC}\left(\rho_{\mathrm{EC}}{ }^{20^{\circ} \mathrm{C}}=0.7886 \mathrm{mg} / \mathrm{mL}^{13}\right)$ to obtain the desired concentration of the dyes in EC. After the addition of DCM to EC, the mixture was stirred and warmed above the melting temperature of the PCM, thus allowing the homogenization of the system. The complete removal of volatile DCM was thus achieved. The samples were transferred into cuvettes to perform the photophysical analysis. In the sample containing both DPA (or, alternatively, DMA or ANT) and PtOEP, the molar ratio between the two dyes was set at 30:1.

Photophysical characterization. The fluorescence emission and excitation spectra have been recorded on a Fluorolog-Spex F112AI spectrofluorometer equipped with a Xenon lamp as an excitation source and a temperature-controlled sample holder. The spectra have been acquired in front face configuration (with a $23^{\circ}$ angle between the excitation light and the detector) and have been corrected for the instrumental response. The emission spectra have been corrected for the self-absorption effect, as reported in ref. 14. Fluorescence decay times were measured by the single photon counting method using an Edinburgh Instrument 199S setup. A $370 \mathrm{~nm}$ nanoLED with a 0.2 ns pulse duration was used as an excitation source and the signal was acquired by a 
Hamamatsu R7400U-03 detector. The decays have been collected using a $45^{\circ}$ configuration between the excitation light and the detector.

\section{Results and Discussion}

Anthracene (ANT) in liquid EC presents well-structured emission and excitation spectra (Figure 1) in agreement with the literature data. ${ }^{15}$ The liquid-to-solid transition of the medium results in dramatic spectral changes. The emission spectrum of ANT in solid EC preserves its vibronic structure, though the relative intensities of the bands are strongly altered and a new broad band centered at $510 \mathrm{~nm}$ appears (Figure 1b). The excitation spectrum loses its characteristic vibronic structure and becomes broadened (Figure 1a). The comparison with literature data supports the assignment of the $510 \mathrm{~nm}$ band to the ANT excimer, ${ }^{16}$ whose formation is also confirmed by the overlap of the excitation spectra recorded at 443 and $510 \mathrm{~nm}$ (Figure S2). The phase change of the medium also affects the fluorescence decay of ANT (Figure S1 and Table S1). The mono-exponential behavior observed in liquid EC ( $\tau=5.1 \mathrm{~ns}$ ) is attributable to the monomeric species, while in the solid sample the decay has a bi-exponential behavior with decay times that vary with the emission wavelength. The shorter decay time for the monomeric emission ( 0.8 and $2.2 \mathrm{~ns}$ at $418 \mathrm{~nm}$ ), was ascribed to the quenching of the excited state due to the formation of excimer species, to which corresponds longer decay times at 510 $\mathrm{nm}(1.4$ and $5.2 \mathrm{~ns})$. Thus, upon EC solidification, the emission spectrum documents that excited state intermolecular interactions are established, although ground state aggregation cannot be excluded to account for the changes in the excitation spectra. Considering the emission maximum of the monomer in liquid eicosane $(3.1 \mathrm{eV})$ and the maximum of the excimer emission 
$(2.4 \mathrm{eV})$, obtained in the solid medium, we conclude that a new emitting state, $0.7 \mathrm{eV}$ downshifted compared to the monomer, raises upon PCM solidification.

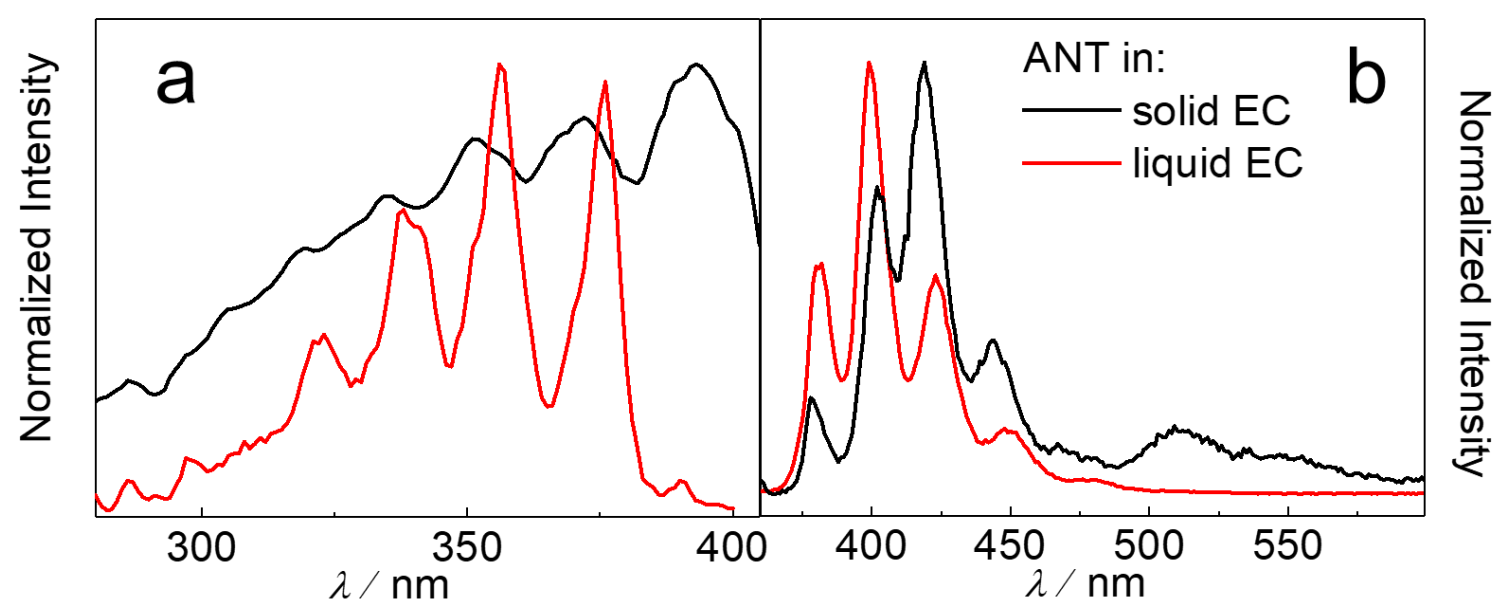

Figure 1. (a) Normalized excitation $\left(\lambda_{\mathrm{em}}=418 \mathrm{~nm}\right)$ and (b) emission spectra $\left(\lambda_{\mathrm{exc}}=326 \mathrm{~nm}\right)$ of ANT $([\mathbf{A N T}]=0.11 \mathrm{mM})$ in liquid and solid EC.

When the emitter has bulky substituents tethered to the anthracene core, as in the case of 9,10 diphenylanthracene (DPA), the liquid to solid transition of EC produces notable but dissimilar changes of the fluorescence behaviour. Once EC solidifies, the excitation spectrum (Figure S3a) broadens and loses the vibronic structure characteristic of monomeric DPA,${ }^{17}$ while the emission spectrum (Figure S3b) is essentially unaltered. In contrast to ANT, this suggests that, although ground-state interactions do take place between DPA molecules in solid EC, ${ }^{18}$ neither excimerlike species formation nor emission energy variation occur. We ascribe these results to the steric hindrance imparted by the large phenyl substituents, which impede achieving sufficiently short interchromophoric distances as to enable for excited state interactions. Actually, the ground state 
aggregation of DPA-analogues has been already observed, ${ }^{18}$ but at fluorophore concentrations at least one order of magnitude higher.

When 9,10-dimethylanthracene (DMA) is used as an emitting unit, both ground-state aggregates and excimers are formed in solid EC. In liquid EC, the structured monomeric emission is observed (Figure 2a) and it has a monoexponential decay whose decay time (10.3 ns) well matches the value reported for DMA in homogeneous solutions. ${ }^{19}$ After cooling the PCM below the $T_{m}$, in addition to the structured emission spectrum $(390-520 \mathrm{~nm})$, the appearance of a broader band centered at $550 \mathrm{~nm}$ is observed (Figure 2a). The latter is due to excimer-like species, ${ }^{20}$ as indicated by the good overlap of the excitation spectra collected at 427 and $540 \mathrm{~nm}$ (Figure 2b). Furthermore, the concomitant broadening of the excitation spectra stands for the presence of ground-state aggregates in the solid medium. In the case of DMA, the energy of the lowest fluorescence state shifts from $2.9 \mathrm{eV}$ to $2.2 \mathrm{eV}$ going from liquid to solid EC.

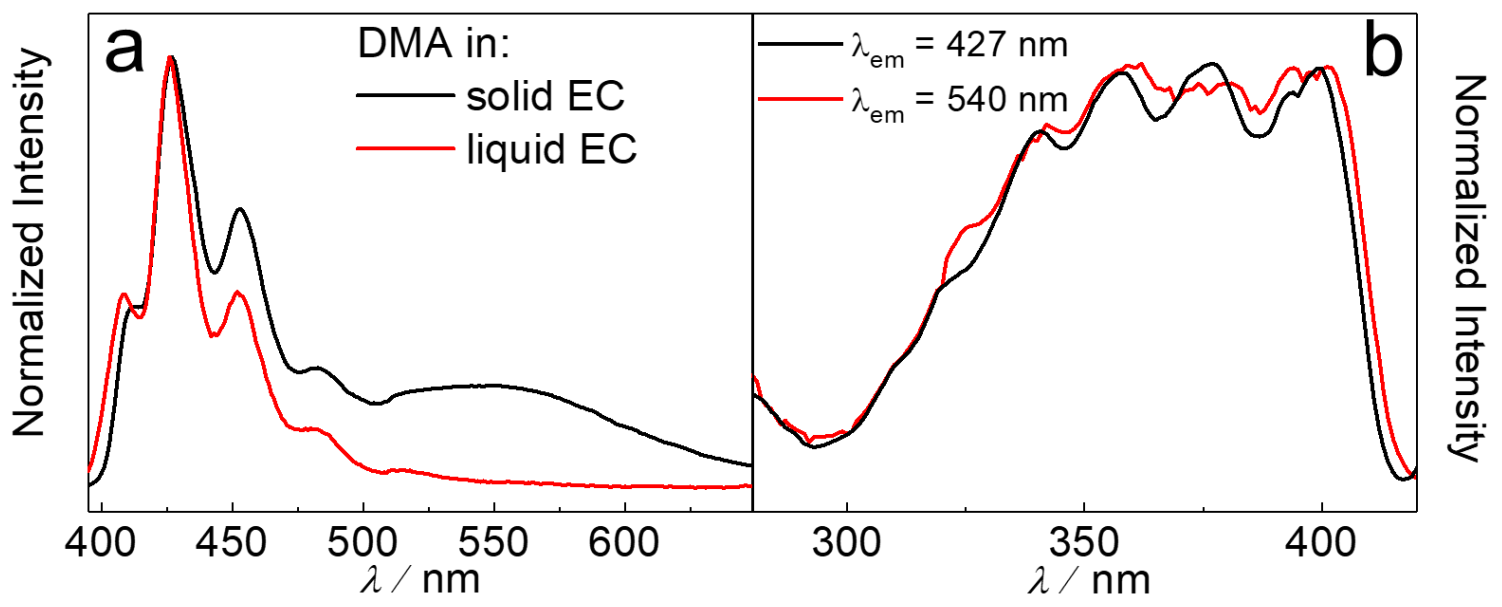

Figure 2. (a) Normalized emission spectra $\left(\lambda_{\mathrm{exc}}=372 \mathrm{~nm}\right)$ of DMA in solid and liquid EC. (b) Normalized excitation spectra recorded at the monomer $\left(\lambda_{\mathrm{em}}=427 \mathrm{~nm}\right)$ and excimer $\left(\lambda_{\mathrm{em}}=540\right.$ $\mathrm{nm})$ emission maxima in solid EC $([\mathbf{D M A}]=1.50 \mathrm{mM})$. 
Time-resolved measurements confirmed that DMA excimer-like species are formed when EC solidifies. Below $\mathrm{T}_{\mathrm{m}}$ the monomer average lifetime decreases significantly $\left(\tau_{\mathrm{mean}}=5.5 \mathrm{~ns}\right)$ and its decay curve could be reproduced by a bi-exponential function (Figure 3, Table S2), confirming the interaction of the excited state, similarly to what observed for ANT. On the other hand, a non-exponential emission decay with much longer average lifetime (53.2 ns) was collected in the excimer region $\left(\lambda_{\mathrm{em}}=560 \mathrm{~nm}\right)$, in agreement with the literature data. ${ }^{21}$

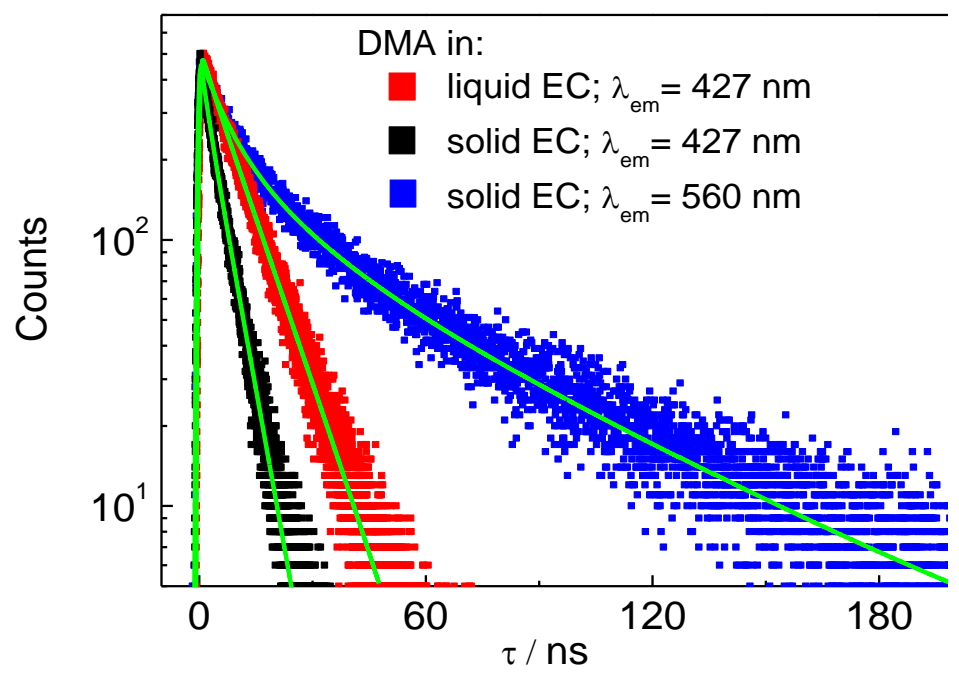

Figure 3. Emission decay curves of DMA in liquid and solid EC. The green lines represent the fitting curves of the experimental points $\left([\mathbf{D M A}]=1.50 \mathrm{mM} ; \lambda_{\mathrm{exc}}=370 \mathrm{~nm}\right)$.

The spectral and decay times modification observed for ANT, DMA and, in a lesser extent, DPA when passing from liquid to solid EC can be attributed to the shortening of the intermolecular distances and to the increase of local fluorophore concentration enabling the occurrence of ground and/or excited state interactions, as already reported for aromatic 
molecules in aliphatic media. ${ }^{22}$ In fact, the PCM liquid-to-solid transition brings spectral modifications analogous to those obtained when increasing the dye concentration (Figure S4-S7).

The presence of other types of chromophores can interfere with the arrangement of the emitting units in the aggregates formed in solid phase EC, thus altering the resulting intermolecular interactions and, as a result, impacting on the energy and kinetic properties of the fluorophore excited states. To verify this hypothesis, we decided to introduce PtOEP (a typical sensitizer for TTA-UC) as an additional chromophore to the anthracene derivatives/EC solutions, choosing a PtOEP/emitter molar concentration ratio (1/30) normally used in TTA-UC systems. ${ }^{23}$

In the presence of PtOEP, the emission spectrum of ANT in solid EC lacks the $510 \mathrm{~nm}$ band (Figure 4a-b), indicating that the formation of excimer-like species is inhibited by addition of the porphyrin molecules. At these conditions, however, the establishment of interactions between PtOEP and the excited state of ANT is confirmed by the shortening of the fluorescence lifetime (quenching) (Figure S8 and Table S3), which reveals that chromophore aggregation does still take place.

The partial structuration of the DPA excitation spectrum and the minor modification of the DPA emission spectrum observed in the presence of PtOEP indicate that porphyrin addition also reduces the DPA ground-state interactions resulting from aggregation (Figure 4c-d). On the other hand, porphyrin effect on the DPA excited singlet manifold is negligible, since the emission spectrum is not modified by the presence of PtOEP. The present data indicate that the efficient UC-emission observed in solid PCM for PtOEP-DPA mixtures ${ }^{7}$ is mainly due to the presence of ground-state heterocomplexes which can facilitate energy transfer processes (e.g. via triplet 
migration). ${ }^{3,4}$ The nature of these complexes and the interaction mechanism deserve deeper investigations.
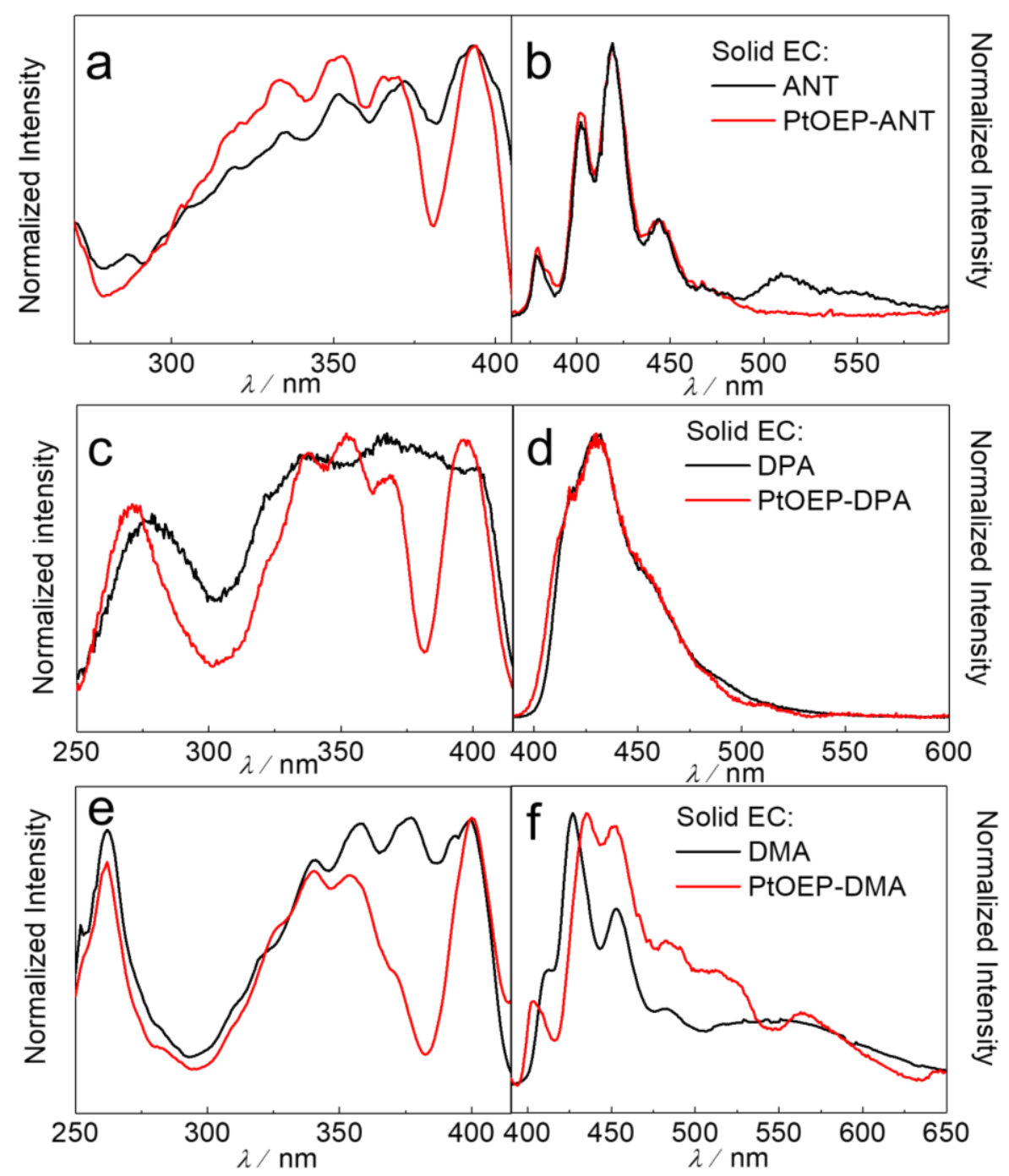

Figure 4. (a) Normalized excitation $\left(\lambda_{\mathrm{em}}=443 \mathrm{~nm}\right)$ and $(\mathrm{b})$ emission $\left(\lambda_{\mathrm{exc}}=326 \mathrm{~nm}\right)$ spectra of ANT and PtOEP-ANT $([$ ANT $]=0.11 \mathrm{mM} ;[\mathrm{PtOEP}]=3.7 \mu \mathrm{M})$. $(\mathrm{c})$ Normalized excitation $\left(\lambda_{\mathrm{em}}\right.$ $=430 \mathrm{~nm})$ and $(\mathrm{d})$ emission spectra $\left(\lambda_{\mathrm{exc}}=372 \mathrm{~nm}\right)$ of DPA and PtOEP-DPA in solid EC $([$ DPA $]=1.35 \mathrm{mM} ;[$ PtOEP $]=45 \mu \mathrm{M})$. (e) Normalized excitation $\left(\lambda_{\mathrm{exc}}=430 \mathrm{~nm}\right)$ and $(\mathrm{f})$ emission $\left(\lambda_{\text {exc }}=372 \mathrm{~nm}\right)$ spectra of DMA in solid EC in the absence and presence of PtOEP 
$([$ DMA $]=1.5 \mathrm{mM} ;[$ PtOEP $]=45 \mu \mathrm{M})$. (The valley at $380 \mathrm{~nm}$ is due to the re-absorption of PtOEP).

The data collected on the PtOEP-DMA system (Figure 4e-f) demonstrate that, in the presence of the porphyrin, both the excimer and the monomer emission bands are preserved, though they appear modified. The presence of PtOEP mainly affects the excited states of the emitter. Decay time measurements (Figure S9, Table S2) performed in the presence of PtOEP reveal that whereas in liquid EC the DMA decay is not affected by the porphyrin, a clear shortening of excimer emission (up to 4 times) in solid EC is observed. This behaviour documents the interactions between the excited DMA (monomer and excimer) and PtOEP, when the dyes are at short distance.

\section{Conclusions}

In summary, we have investigated the fluorescence behaviour of three anthracene derivatives to rationalize the impact of medium phase change on the interchromophoric ground and excited state electronic interactions. While in the liquid state the dyes all show the monomeric fluorescence features, indicating lack of intermolecular interactions, in the solid state, these interactions are established at low dye concentrations $(\leq \mathrm{mM})$. The electronic state involved depends on the substituents: ground-state aggregates are observed for DPA while both groundstate aggregates and excimers are detected for ANT and DMA, with the latter forming excimers predominantly. In the latter case, the formation of excimer-like species results in changes in the energy of the emitting state of about $0.7 \mathrm{eV}$ and this shift is preserved even in the presence of an interfering dye. We demonstrate that by simply changing the temperature from above to below 
the melting point of PCM the tuning between monomer to excimer (and/or aggregate) emission can be produced.

Supporting Information. The Supporting Information, containing supplementary figures, is available free of charge on http://pubs.acs.org

\section{AUTHOR INFORMATION}

Corresponding authors:

e-mail: loredana.latterini@unipg.it; croscini@icn2.cat

ORCID:

Loredana Latterini - 0000-0002-1021-9423

Claudio Roscini - 0000-0002-0157-8934

Daniel Ruiz Molina - 0000-0002-6844-8421

Giuseppina Massaro - 0000-0002-3971-8453

\section{Notes}

The authors declare no competing financial interests.

\section{ACKNOWLEDGMENT}

The University of Perugia and the Ministero Istruzione dell'Università e della Ricerca (MIUR) are thanked for the financial support through the program "Dipartimenti di Eccellenza 20182022" (grant AMIS).

This work was supported by project MAT2015-70615-R and CTQ2015-65439-R from the Spanish Government and by FEDER funds. ICN2 acknowledges support from the Severo Ochoa 
Program (MINECO, Grant SEV-2013-0295). Funded by the CERCA Programme/Generalitat de Catalunya.

\section{REFERENCES}

(1) Kasha, M. Energy transfer mechanisms and the molecular exciton model for molecular aggregates Radiat. Res. 1963, 20, 55-70.

(2) Hestand, N. J.; Spano, F. C. Molecular aggregate photophysics beyond the Kasha model: Novel design principles for organic materials Acc. Chem. Res. 2017, 50, 341-350.

(3) Goudarzi, H.; Keivanidis, P. E. All-Solution-Based Aggregation Control in Solid-State Photon Upconverting Organic Model Composites ACS Appl. Mater. Interfaces 2017, 9, $845-857$.

(4) Pengfei, D.; Yanai, N.; Kurashige, Y.; Kimizuka, N. Aggregation-Induced Photon Upconversion through Control of the Triplet Energy Landscapes of the Solution and Solid States Angew. Chem. Int. Ed. 2015, 54, 7544-7549.

(5) Singh-Rachford, T. N.; Lott, J.; Weder, C.; Castellano, F. N. Influence of Temperature on Low-Power Upconversion in Rubbery Polymer Blends J. Am. Chem. Soc. 2009, 131, $12007-12014$.

(6) Massaro, G.; Gentili, P. L.; Ambrogi, V.; Nocchetti, M.; Marmottini, F.; Ortica, F.; Latterini, L. Triplet-triplet annihilation based upconversion in silica matrices. Microp. Mesop. Mat. 2017, 246, 120-129.

(7) Massaro, G.; Hernando, J.; Molina, D.R.; Roscini, C.; Latterini, L. Thermally Switchable Molecular Upconversion Emission. Chem. Mater. 2016, 28, 738-745.

(8) Langelaar, J.; Jansen, G.; Reitschnick, R. P. H.; Hoytlnk, G. J. On the lifetime of triplet monomer and excimer of aromatic hydrocarbons in solutions. Chem. Phys. Lett. 1971, 12, $86-91$. 
(9) Chandra, A. K.; Lim, E. C. Semiempirical Theory of Excimer Luminescence. II. Comparison with Previous Theories and Consideration of the Transition Probability and the Stability of the Excimer Triplet State. J. Chem. Phys. 1968, 49, 5066-5072.

(10) East, A. L. L.; Lim, E. C. Naphthalene dimer: electronic states, excimers, and triplet decay. J. Phys. Chem. 2000, 113, 8981-8994.

(11) Pabst, M.; Lunkenheimer, B.; Kohn, A. The Triplet Excimer of Naphthalene: A Model System for Triplet-Triplet Interactions and Its Spectral Properties. J. Phys. Chem. C 2011, $115,8335-8344$.

(12) Lide, R. D. CRC Handbook of Chemistry and Physics [Online]; CRC Press: Boca Raton, FL, 2005; p 374, http://hbcponline.com (accessed July 18, 2018).

(13) Haynes, W.M. (ed.). CRC Handbook of Chemistry and Physics. 95th Edition. CRC Press LLC, Boca Raton: FL 2014-2015, p. 3-240.

(14) Gentili, P.L., Clementi, C., Romani, A. Appl. Spectrosc. 2010, 64, 923-929; Ultraviolet-Visible Absorption and Luminescence Properties of Quinacridone-Barium Sulfate Solid Mixtures.

(15) Montalti, M.; Credi, A.; Prodi, L.; Gandolfi, M. T. Handbook of photochemistry, 2006, 3rd edn. CRC Press, Boca Raton.

(16) McVey, J.K.; Shold, D.M.; Yang, N.C. Direct observation and characterization of anthracene excimer in solution J. Chem. Phys. 1976, 65, 3375.

(17) Hamal, S.; Hirayama, F. Actinometric Determination of Absolute Fluorescence Quantum Yields. J. Phys. Chem. 1983, 87, 83-89.

(18) Oyamada, T.; Akiyama, S.; Yahiro, M.; Saigou, M.; Shiro, M.; Sasabe, H.; Adachi, C. Unusual photoluminescence characteristics of tetraphenylpyrene (TPPy) in various aggregated morphologies. Chem. Phys. Lett. 2006, 421, 295-299. 
(19) Scully, A. D.; Yasuda, H.; Okamoto, M.; Hirayama, S. The effect of pressure on the dynamic quenching by oxygen of the excited singlet state of 9, 10-dimethylanthracene in solution. Chem. Phys. 1991, 157, $271-278$.

(20) Birks, J. B. Excimers. Rep. Prog. Phys. 1975, 38, 903-974.

(21) Mataga, N.; Torihashi, Y.; Ota, Y. Studies on the fluorescence decay times of anthracene and perylene excimers in rigid matrices at low temperatures in relation to the structures of excimers Chem. Phys. Lett. 1967, 1, 385-387

(22) Duan, P.; Yanai, N.; Nagatomi, H.; Kimizuka, N. Photon Upconversion in Supramolecular Gel Matrixes: Spontaneous Accumulation of Light-Harvesting Donor-Acceptor Arrays in Nanofibers and Acquired Air Stability. J. Am. Chem. Soc. 2015, 137, 1887-1894;

(23) Penconi, M.; Ortica, F.; Elisei, F.; Gentili, P.L. New molecular pairs for low power noncoherent triplet-triplet annihilation based upconversion: dependence on the triplet energies of sensitizer and emitter. J. Lumin. 2013, 135, 265-270 


\section{TOC GRAPHICS}

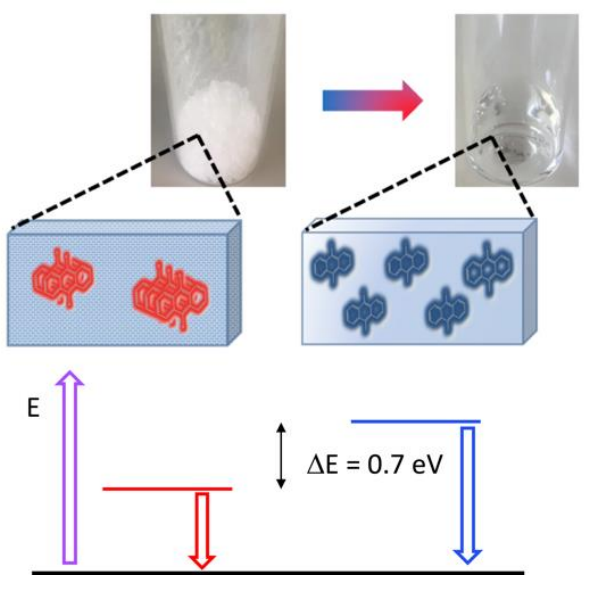

